\title{
Analysis of Nonlinear Phenomena in Industry University Research Cooperation Innovation System
}

\author{
Wei Jiang ${ }^{1,2}$ \\ ${ }^{1}$ Fundamental Science on Radioactive Geology and Exploration Technology Laboratory, East China \\ University of Technology, Nanchang, Jiangxi, China \\ ${ }^{2}$ Key Laboratory of Innovation Method and Decision Management System of Guangdong Province, \\ South China University of Technology, Guangzhou, Guangdong, China \\ jw186@163.com
}

Keywords: Cooperation; Innovation; Nonlinear

\begin{abstract}
This paper analyzed nonlinear phenomena in industry university research cooperation innovation system. It was pointed out that the industry university cooperative innovation system has nonlinear phenomena and the cooperative innovation behavior is a complex dynamic system. The cooperative innovation system has the characteristics of insect breeding system. It can construct with Logistic model to simulate the system. The research has an important theoretical and practical significance to provide effective decision support for government.
\end{abstract}

\section{Introduction}

The cooperative innovation system is several relatively independent entities by enterprises, universities, research institutions and government, all subjects have different forms of organization, cooperative innovation can only be done by the relatively independent entity coordination. Each subject has its own interests and management, and there is no relationship between the principal and the slave. The change of any subject itself will affect the change of other subjects, and the whole system can continuously study and reorganize and perfect its hierarchical structure and function structure. There are many interrelated factors in industry university research cooperation innovation system, which are interrelated and crisscross. Under the combined effect of these complex characteristics, the cooperative innovation behavior of Industry University Research Institute shows strong complexity characteristics.

Based on the complexity analysis of the phenomenon of cooperative innovation, forms of existence and the changes and study many problems of the function mechanism of the cooperative innovation research attempts to explore the inherent law and external effects, establish a theoretical system of cooperative innovation behavior research complex system, work can be beneficial to guide cooperation innovation behavior, motivation and normative role, with increasingly complex problems can help to solve the cooperative innovation in the process of facing, accelerate the cooperative innovation process, but also for the amendment, supplement and perfect the research of cooperative innovation theory system has been established[1-4].

\section{Analysis of Nonlinear Phenomena in Industry University Cooperation Innovation System}

The cooperative innovation behavior is a complex dynamic system, and it can realize the complexity analysis of industry university research cooperation innovation system. The interaction and interdependence among the factors in the research cooperation innovation is the important prerequisite for the complexity of the system. The complexity of the software first behavior of cooperative innovation behavior is defined, and then construct a dynamic complex system model, from a system dynamics perspective on the cooperative innovation behavior research system, reveals the dynamic evolution path and system complexity of cooperative innovation research[5-6]. 
The system of industry university research cooperation is affected by the internal factors of the system and the interference of external factors. Under the circumstances, the operating track of the system changes greatly, which makes the system finally produce unexpected results. The cooperative innovation system has the characteristics of insect breeding system, continue to occur with generational changes, generations continue to copy and repetition of the previous generation gene, constructed with Logistic population model to simulate the system, continuous breeding between generations. There are similarities and differences between the offspring and the previous generations[7].

The Logistic model is used to analyze the cooperative innovation system of Industry University Research Institute, and the period doubling bifurcation model and the insect model of Logistic regression analysis are consistent with the basic conditions of the cooperative innovation system of industry university research institute. First, the insect population $\mathrm{n}$ generation to simulate the cooperative innovation system of $n$ generation, $n-1$ generation with the insect population to simulate the cooperative innovation system of the $n-1$ generation, to simulate the cooperative innovation system with the insect population environment for the survival of the external environment, the cooperation through continuous upgrading constantly updated innovation system as the existence of absorption the advantages of the system and reflect on the problems in system on the basis of the previous generation.Generation by generation, innovative ways of inheritance,like insect populations, survive the natural laws of external influences and their own racial interference, the experience and virtues of the previous generation being absorbed. Through continuous inheritance between generations with self similarity evolution, and this evolution has prompted the next generation of insects can survive in a difficult environment, facing the interference of environment under the new, will inherit the dominant gene. Therefore, the evolutionary mode between this generation and generation is escalating as the industry university cooperative innovation system, showing the mode of replication between the offspring and the father in the chaotic phenomenon. To sum up, it can confirm that the research institute cooperative innovation system conforms to the conditions of the pest model, and can use the model to study and describe the chaotic phenomena of industry university research cooperation innovation system[8].

The formula model of image, $x_{n}$ and $x_{n+1}$ of the mathematical relationship between the reaction in the axis is a parabola, look up through the picture when $x_{n+1}=x_{n}$ 's cooperative innovation system is in the stable state. The selection of a zero line to analyze the population model of cooperative innovation system, with a linear $x_{n+1}=x_{n}$ and parabolic $x_{n+1}=\lambda x_{n}\left(1-x_{n}\right)$, so this situation there are two categories: the first category is parabolic in the line below, parabola and straight lines do not exist second points, that is to say the generational renewal is stable and the interference system from internal and external; then the second class is part of a parabola straight line, parabola and straight lines are second points that the cooperative innovation system is not disturbed.

Through the analysis of the above two kinds of circumstances, can be an important point in the system, which is the linear tangent position of parabola, $x_{n}=0$ and $\lambda=1$ can be deduced. So when $\lambda<1$, in the range $x_{n+1}>0$, there is only one intersection between the parabola and a 45 degree line, and this point is the origin, as shown in Fig.1, when $x_{n}=0$ is the final stable point of cooperative system, namely cooperative innovation system in internal and external interference factors, the system is still in its original state, which means that the cooperative innovation system development to the final system will face the loss of vitality of the phenomenon. So the conclusion is that when the factors of the internal and external environment and system changes, system changes in these factors are still not changed, a power system may be in effect factors, the system still maintain the status quo, but when the time is long past, due to the loss of power, will be gradually eliminated by the market and failure. 


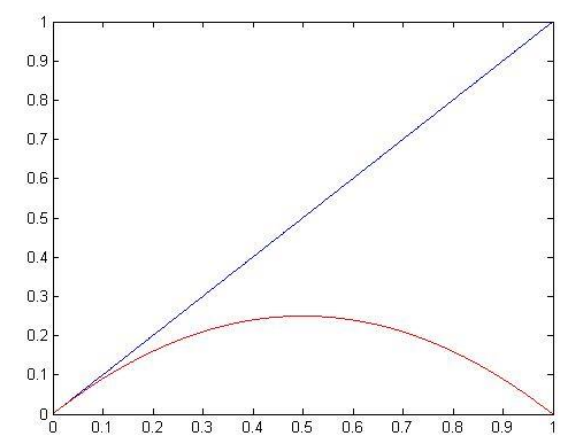

Figure 1. $x_{n}=0$ Iteration diagram

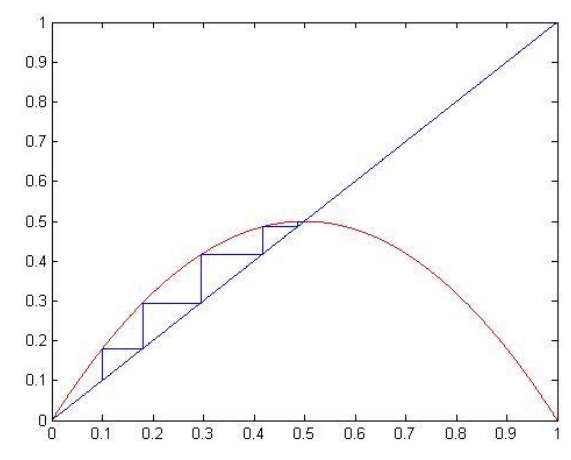

Figure 2. $3>\lambda>1$ Iteration diagram

When $3>\lambda>1$, the public number of cooperative innovation system in image slash and parabola increased from 1 to 2 as shown in Fig.2, the number of cycles at 1 . In this case, the stable point of industry university research cooperation innovation has changed from original origin to $1-1 / \lambda$. When the $\lambda$ value increases, the farther away from the intersection of $1-1 / \lambda$ and the origin that, when the system of internal and external factors of the system increases, the system is more far away from the equilibrium state, the influence factors into a moderate form of incentive, cooperative innovation system appears to another steady development trend and is different from the original the state, the system exhibits a positive attitude. On the contrary, when the value of $\lambda$ decreases when the $1-1 / \lambda$ value is smaller, the said system deviating from the original equilibrium state is small, when the $1-1 / \lambda$ value is close to the origin of the state, said more and far from equilibrium system is not obvious. The positive incentive effect of the system is less obvious. In this case, the starting point is that no matter where the cooperative innovation, cooperative innovation system to promote the development and evolution of the system in the internal and external factors, the system will tend to be stable in the $1-1 / \lambda$ position, and then wait for the next development system or directly to another the advantages and development status.

When 3.569946> $>>3$, the iteration conditions as shown in Fig.3, the original position of both systems in what place, as long as the right to control the value of $\lambda$ can ensure the cooperative innovation system under the influence of $\lambda$, enhance the cooperative innovation system, making the system more perfect, between the various elements of the system and each subsystem contact more closely, but also to strengthen the enterprises, research institutions and the government in the face of changes in the external market environment also can have enough competitiveness.

When $3.569946>\lambda>3$, the intersection of $1-1 / \lambda$ will not stable, cooperative innovation system will be a change to the multi periodic solutions, periodic solutions, namely now system of internal and external environmental factors in the system of the incentive effect of some strong stimulation, which makes the system deviate from the original state of the system is acceptable from the edge of the state, the system restore balance the difficulty is too big.

So in the $3.569946>\lambda>3$ state, the incentive effect of the system of internal and external environment factors of the system is still valid, but as the cooperative innovation of such a system, the incentive effect is too strong too cause the system out of equilibrium, makes the system to another balance state, reduce the self similarity with the original system the same as the $3.569946>\lambda>3$ case, reflect the manufacture learning research cooperation and innovation in the choice of starting point is not at the same time, the characteristics of evolution may eventually appear different end point, but the system still to another equilibrium.

When $4>\lambda>3.569946$, the industry university research cooperation innovation system will enter the chaotic state as shown in Fig. 4. At this time, excessive stimulation system of internal and external environmental factors for the performance incentive system, performance is due to these factors have impact on the system makes the system excessive deviation from the equilibrium state, the interaction of the elements in the system is too fierce, that in spite of these factors, the system is indeed in the ability to restore equilibrium, the cooperative innovation system, mutual communication between 
enterprises, government and academic research institutions too closely, the boundaries between government and enterprises, the research institutions are no longer so clear, function confusion. This chaos there may be a small detail, not handled properly will result in the butterfly effect system, cooperative innovation system of internal and external environment of a small initial change will completely change the system track end point. At the same time, it is difficult to control the system effectively and the system is out of control risk.

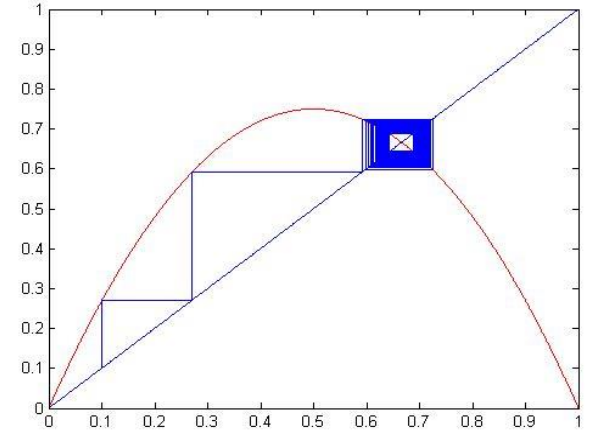

Figure 3. 3.569946 $>\lambda>3$ Iteration diagram Figure 4. $4>\lambda>3.569946$ Iteration diagram which makes the system into chaos, if the population model even together, then we can explain the bifurcation to the image of the integrity of the implementation process.

When the influence factors of cooperative innovation system makes the system appear in the 3 cycle segmentation bifurcation point, and then ended in 3.569946, when the system factors in the range of $3 \sim 3.569946$, the system development of the replication system and re generation, and the effect of the amplification of the resulting system breaks the original state of development to another equilibrium state in the development of. But when the system is in the range of 3.569946 4, the influencing factors for the incentive system is excessive, makes the system not to have the direction of the future development of the system, the randomness of the jump, in this state, the system is easy to lose control, as shown in Fig. 5.

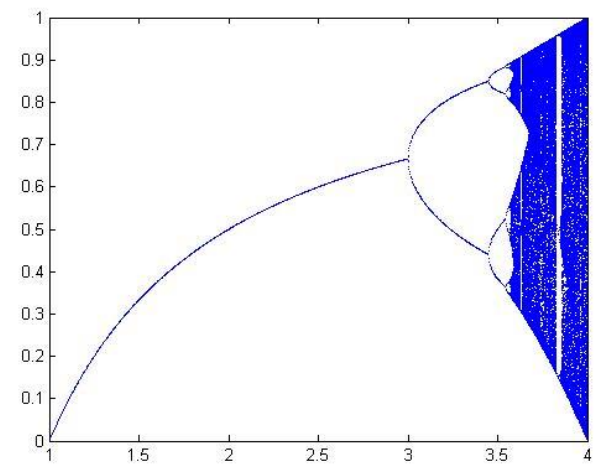

Figure 5. Bifurcation diagram of collaborative innovation

The system of cooperative innovation research in complexity will find similar sudden shock phenomenon, namely a variation of parameters of nonlinear dynamics system, with time system with intermittent movement rules of motion component is irregular, the irregular intermittent movement will be accompanied by the change of parameters increased, the impact of this sudden more and more strong, and finally into the chaotic state, the conversion from intermittent state to chaotic phenomenon known as intermittency.

\section{Summary}

Under the interaction of system internal and external environment, the influencing factors of system development is $\lambda=f\left(s_{1}, s_{2}, s_{3}, \Lambda\right)$, and its different values will make the collaborative innovation 
system of Industry University Research Institute develop differently. First of all, $s_{1}, s_{2}$ and $s_{3}$ indicate that the intensity of interaction between the elements of enterprises, government and academic institutions will influence the factors that influence the development of the system. Secondly, $\Lambda$ indicates another influence factor of external environment on R \& D Cooperative Innovation system. Therefore, the reasonable control system of the factors affecting the development of $\lambda$, namely, enterprises and research institutions cooperation should not exceed the cooperation and cooperation in the blind, cooperative innovation system, completes the enterprise product and research direction of match, at the same time the enterprise technology transformation can meet the research institutions the ability of technological innovation, both complementary advantages and common progress, and as the leading cooperative innovation system, the government should make laws and regulations and policies in the system development, internal environment can not promote the system development and forced change system, combined with the laws of the market, in the development of power system tends to weaken, the government macro-control, to maintain the development momentum in the system, and find new ways in the continuous development of the system.

\section{Acknowledgements}

The soft science research project of Jiangxi province (20151BBA10053) and college of humanities and social science research projects in Jiangxi province (GL1507) and technology research project of Jiangxi education department (GJJ151534) (GJJ151543) and Jiangxi education planning project (15YB041) and open fund of fundamental science on radioactive geology and exploration technology laboratory (No. RGET1511) supported this work.

\section{References}

[1] LIU Youjin, LIU Lijun. Study on the evolement processionof the cluster innovation netwoek based on the chaos theory. Studies in Science of Science. 2 (2008) 185-190.

[2] C.Fuchs, W.Hofkirchner, Self-organization, knowledge and responsibility. Kybernetes.2 (2005) 241-260.

[3] R.C. Calia, FM Guerrini, GL Moura, Innovation networks: From technological development to business model reconfiguration. Technovation. 8 (2007) 426-432.

[4] A. Bergek, S. Jacobsson, B Carlsson, S Lindmark, Analyzing the functional dynamics of technological innovation systems: A scheme of analysis. Research Policy. 3 (2008)407-429.

[5] LI Wenbo, ZHANG Wenzhe. On the complexity of enterprise integrated innovation system: chaos and fractal. Studies in Science of Science.4(2006)618-623.

[6] O.N. Boldov, Innovation dynamics and financial markets in developed countries from a self-organization perspective. Studies on Russian Economic Development. 5(2008)523-530.

[7] DANG Xing-hua, ZHENG Deng-pan, The Self-organization Evolution Model of Modular Technology Innovation Network. R\& D MANAGEMENT. 4 (2009)54-59.

[8] Ma Fei-hong, University-Industry-Government Cooperative Innovation System Modeling and Simulation Method Research. Computer Simulation. 9 (2012) 1-5. 